\title{
COMPOSICIÓN, ESTRUCTURA Y DIVERSIDAD DE LA COMUNIDAD DE ÁCAROS MESOSTIGMATA DE UN HAYEDO NATURAL (FAGUS SYLVATICA) DEL SUR DE EUROPA
}

\author{
M. L. Moraza
}

\begin{abstract}
RESUMEN
Se estudia la composición específica de la comunidad de ácaros Mesostigmata en una hayedo (Fagus sylvatica L.) de Navarra (España, Sur de Europa). Se han identificado 653 ácaros procedentes de 12 muestras. Estos representan a 41 especies de 14 familias y las especies más abundantes son Paragamasus ponantinus Athias-Henriot, 1967; Vegaia nemorensis (C. L. Koch, 1839); Paragamasus rothamstedensis Bhattacharyya, 1963 y Rhodacarus coronatus Berlese, 1921. La mitad de la comunidad habita en el horizonte húmico del suelo. En este hayedo el valor de la diversidad de Shannon $\left(H^{\prime}\right.$ $\log _{2}$ ) es 4,42 y el de la equitabilidad $\left(J^{\prime}\right) 0,82$.
\end{abstract}

Palabras clave: Acari, Mesostigmata, comunidad, diversidad, hayedo, Navarra, España.

\section{ABSTRACT \\ Species composition, structure and diversity of the soil Mesostigmata mite community in a natural beech forest (Fagus sylvatica) from southern Europe}

Species composition, structure and diversity of the soil Mesostigmatid mite's community was studied in European beech forest (Fagus sylvatica L.) in Navarra (Spain, southern Europe). Twelve samples were taken and 653 mites were identified. They represented to 41 species from 14 families, the most abundant species being Paragamasus ponantinus Athias-Henriot, 1967; Veigaia nemorensis (C. L. Koch, 1839); Paragamasus rothamstedensis Bhattacharyya, 1963 and Rhodacarus coronatus Berlese, 1921. Haft part of the mites community inhabits the humic layer of the soil. The value of the Shannon's diversity index $\left(\mathrm{H}^{\prime} \log _{2}\right)$ in the forest studied is 4,42 , and the equitability $\left(J^{\prime}\right)$ is 0,82 .

Keywords: Acari, Mesostigmata, community, diversity, beech forest, Navarra, Spain.

* Departamento de Zoología y Ecología, Facultad de Ciencias, Universidad de Navarra, C/ Irunlarrea s/n, Pamplona 31080 (Navarra), España, Tel: 3448 425600; Fax: 3448 425649; e-mail: mlmoraza@unav.es 


\section{Introducción}

Los hayedos navarros son los más meridionales de Europa, exceptuando algunos núcleos o plantas aisladas sobre suelos silíceos en la Cordillera Ibérica (Sistema Central) en las caras norte de los montes. En esta región son bosques sometidos a entresacas y/o talas parciales o totales que los convierten en vastas praderas o bien son parcialmente sustituidos por pequeñas extensiones de alerce (Larix kaempheri). Los ácaros oribátidos de estos hayedos han sido previamente estudiados (Moraza et al., 1981; Moraza, 1982) junto con las comunidades de colémbolos y nematodos (Arbea \& Jordana, 1988; Ariño et al., 1992). Los mesostigmata son ácaros depredadores a diferencia de los anteriores y sus comunidades en este tipo de bosque no han sido específicamente estudiadas en esta y otras regiones europeas de la misma manera que lo han sido en robledales, pinares y otros medios (Usher, 1971; Buryn, 1992; Ageeva, 1995; Salmane, 2000). Su conocimiento es también indispensable para comprender y evaluar el efecto que los cambios ambientales, incluido cualquier tipo de perturbación humana (reforestación, entresaca, tala, incendios...), puedan ocasionar en los hábitat naturales. Es por esto que el presente trabajo pretende ser una contribución general al conocimiento de estos ácaros en los hayedos sur europeos y contribuir al conocimiento de la diversidad faunística de la Península Ibérica en particular.

Este trabajo es parte del contrato de investigación $\mathrm{N}^{\mathrm{o}} .0220$ del CAICYT que lleva por título "Efecto de la explotación y repoblación forestal sobre la fauna del suelo". El estudio de los ácaros Mesostigmata de este proyecto ha sido además financiado por el Departamento de Educación, Universidades e Investigación del Gobierno Vasco (España), y The Acarology Laboratory (Departmen of Entomology) The Ohio State University (USA).

\section{Material y métodos}

LOCALIDAD ESTUDIADA. El hayedo de Irati se desarrolla sobre un sustrato constituido por calizas, dolomías, calizas arcillosas, margo-calizas y margas del Terciario. Esta ubicado en una zona donde predomina la serie de vegetación pirenaica basófila y ombrófila Scillo lilio-hyacinthi-Fageto sylvaticae Sygmetum, que se asienta sobre Typic Dystrudept. Una de las características del balance hídrico del suelo de este hayedo es que el periodo de utilización del agua almacenada se limita prác- ticamente al mes de julio, volviendo a estar el suelo saturado antes de que finalice el verano (Amores, 2002). Las características fisiográficas de este hayedo son: latitud (N): 42 ${ }^{\circ} 58^{\prime} 650^{\prime \prime}$; longitud (O): $01^{\circ} 06^{\prime} 353^{\prime \prime}$; altitud (m): 1.250; Temperatura media anual histórica $\left({ }^{\circ} \mathrm{C}\right): 6,6$; precipitación media anual histórica (mm): 1.999; evapotranspiración potencial (ETP) $(\mathrm{mm})$ : 538; orientación NO; pendiente $\left({ }^{\circ}\right)$ : 10 ; coordenadas UTM: 30TXN56.

MUESTREO. El muestreo de realizó una vez en cada estación climática, en los meses de Mayo, Agosto, Octubre y Enero (08/VI/1982; 18/VIII/1982; 03/X/1982 y 18/I/1983). Se muestreó una única superficie de $25 \times 25 \mathrm{~cm}$. separándose tres horizontes o capas del suelo: la hojarasca (Aoo), la capa húmica $(\mathrm{H})$ y la capa mineralizada $(\mathrm{B})$. La profundidad de la muestra dependía de la cantidad de hojarasca en el suelo. De esta forma se obtuvieron un total de 12 muestras (4 de hojarasca, 4 de humus y 4 de suelo mineral).

Junto con las muestras se tomaron algunos parámetros físico-químicos del suelo, tanto en superficie como en profundidad (Tabla 4). El pH se midió en agua destilada y la humedad del suelo (\% de agua) se calculó sustrayendo al peso fresco de la muestra el peso de esta tras haber sido desecada a $20^{\circ} \mathrm{C}$

MÉTODO DE EXTRACCIÓN. La mesofauna se extrajo de las muestras usando el método de Tullgren y los ácaros se examinaron con un microscopio óptico provisto de contraste de fases previo su aclarado con líquido de Nesbitt y montaje en medio de Hoyer.

ESTADÍSTICOS. Los índices de diversidad de las comunidades se calcularon para cada lote de muestras estacionales (sumando los ejemplares de las tres capas del suelo) para realizar comparaciones globales y para la muestra final producto de la suma de las muestras estacionales y considerada como representativa de la comunidad en ese medio.

Los ejemplares que no pudieron ser identificados a nivel de especie por tratarse de estadios inmaduros de géneros representados por más de una especie, son considerados como pertenecientes a una misma especie en los análisis de diversidad.

La abundancia relativa de la especie es el número de ejemplares de la especie $\left(N_{i}\right)$ en relación al número total de ejemplares $\left(N_{t}\right)$ encontrados en la muestra $\left[\left(\mathrm{A}=N_{i} / N_{t}\right) \times 100\right]$. 
Tabla 1. - Listado de especies ordenadas de acuerdo con la abundancia relativa de sus poblaciones (A \%), con indicación del número de ejemplares de cada estadio de desarrollo y sexo de los adultos: distribución vertical en el suelo (Aoo: hojarasca; H: mantillo; B: suelo mineralizado). Las especies precedidas de un asterisco son nuevas citas para la fauna ibérica.

Table 1.- List of species organized according to decreasing overall relative abundance (A); number of specimens at all development stages and sex of adults; vertical distribution in soil (Aoo: leaf litter; H: humus; B: mineral soil). Species marked by an asterisk are new citations for the Iberian fauna.

\begin{tabular}{|c|c|c|c|c|c|c|c|c|c|c|}
\hline MESOSTIGMATA & ㅇ & $0^{x}$ & D & $\mathbf{P}$ & $\mathbf{L}$ & Total & A \% & Aoo & $\mathbf{H}$ & B \\
\hline Parasitidae inmature & & & & -120 & & 100 & 18,1 & + & + & + \\
\hline *Paragamasus ponantinus Athias-Henriot, 1967 & 28 & 27 & & & & 55 & 8,3 & - & + & + \\
\hline Veigaia nemorensis (C. L. Koch, 1839) & 23 & 1 & 25 & 1 & & 50 & 7,5 & + & + & + \\
\hline Paragamasus navarrensis Athias-Henriot, 1977 & 15 & 12 & 13 & 1 & & 41 & 6,2 & + & + & + \\
\hline *Paragamasus rothamstedensis Bhattacharyya, 1963 & 25 & 14 & & & & 39 & 5,9 & + & + & + \\
\hline Rhodacarus coronatus Berlese, 1921 & 38 & & & & & 38 & 5,7 & + & + & + \\
\hline *Pergamasus crassipes (Linnaeus, 1758) & 8 & 6 & 16 & 4 & & 33 & 5,0 & + & + & + \\
\hline *Trachytes welbourni Moraza, 1989 & 9 & 9 & 8 & 2 & & 28 & 4,2 & + & + & + \\
\hline Polyaspinus cylindricus Berlese, 1916 & 11 & & 2 & 10 & 2 & 25 & 3,8 & + & + & + \\
\hline Cilliba cassidea (Hermann, 1804) & & 3 & 9 & 4 & 8 & 24 & 3,6 & + & + & + \\
\hline Prozercon davidi Moraza, 2006 & 12 & 7 & 2 & 1 & & 22 & 3,3 & - & - & + \\
\hline Veigaia planicola (Berlese, 1892) & 7 & 2 & 12 & & & 21 & 3,2 & + & + & + \\
\hline *Zercon arcuatus Trägårdh 1931 & 11 & 3 & 5 & & 2 & 20 & 3,0 & + & + & + \\
\hline *Parasitus kraepelini Karg, 1971 & 14 & 3 & 1 & 1 & 1 & 20 & 3,0 & + & + & - \\
\hline Arctoseius minutus (Halbert, 1915) & 14 & 2 & & & & 16 & 2,4 & - & + & - \\
\hline *Holoparasitus stramenti c.f. Karg, 1971 & 8 & 5 & 2 & & & 15 & 2,3 & + & + & - \\
\hline Parasitus sp. 1 & 6 & 1 & 3 & & & 10 & 1,5 & + & + & - \\
\hline *Uropoda misella (Berlese, 1916) & 2 & 2 & 6 & & & 10 & 1,5 & - & + & - \\
\hline Pachyseiulus ignacii Moraza \& Johnston & 4 & 4 & & & & 8 & 1,2 & - & + & - \\
\hline Pergamasus robustus (Oudemans, 1902) & 1 & 7 & & & & 8 & 1,2 & - & + & + \\
\hline *Zercon parivus Moraza, 1991 & 6 & 2 & & & & 8 & 1,2 & - & + & - \\
\hline *Orthadenella lawrencei (Evans, 1957) & 4 & 2 & & & & 6 & 0,9 & + & + & - \\
\hline Pachyseius morenoi Moraza & 6 & & & & & 6 & 0,9 & + & + & - \\
\hline *Arctoseius venustulus (Berlese, 1907) & 3 & 1 & & & & 4 & 0,6 & + & + & - \\
\hline Paragamasus sp. 1 & 4 & & & & & 4 & 0,6 & - & + & + \\
\hline Paragamasus sp. 2 & 2 & 1 & 1 & & & 4 & 0,6 & + & - & - \\
\hline *Dinychus hispanicus (Gilyarov \& Bregetova, 1977) & & & 1 & 2 & & 3 & 0,5 & - & + & - \\
\hline *Macrocheles montanus (Willmann, 1951) & & 1 & 2 & & & 3 & 0,5 & + & + & - \\
\hline Pseudolaelaps doderoi Solari, 1954 & 3 & & & & & 3 & 0,5 & - & - & + \\
\hline Uropodina sp. 1 & & 1 & 2 & & & 3 & 0,5 & + & + & - \\
\hline *Holoparasitus calcaratus (C. L. Koch, 1839) & 2 & & & & & 2 & 0,3 & - & - & + \\
\hline *Pachylaelaps longisetis Halbert, 1915 & 1 & 1 & & & & 2 & 0,3 & - & + & + \\
\hline Paragamasus sp. 3 & 2 & & & & & 2 & 0,2 & - & + & - \\
\hline Pergamasus sp. & & 1 & 1 & & & 2 & 0,3 & - & + & + \\
\hline Zercon guadarramicus Mihelcic, 1960 & 1 & 1 & & & & 2 & 0,3 & - & + & - \\
\hline *Anthoseius pirianykae Wainstein 1972 & 1 & & & & & 1 & 0,2 & + & - & - \\
\hline Macrolaspis sp. & 1 & & & & & 1 & 0,2 & - & + & - \\
\hline *Poecilochirus necrophori Vitzthum, 1930 & & & 1 & & & 1 & 0,2 & + & - & - \\
\hline *Proctolaelaps robustus Evans, 1958 & 1 & & & & & 1 & 0,2 & + & - & - \\
\hline Trachytes aegrota (C. L. Koch, 1841) & 1 & & & & & 1 & 0,2 & - & - & + \\
\hline *Veigaia sanamamedi Athias-Henriot, 1961 & 1 & & & & & 1 & 0,2 & - & + & - \\
\hline Zercoseius spathuliger (Leonardi, 1889) & & 1 & & & & 1 & 0,2 & - & - & + \\
\hline Número total de ejemplares & 269 & 115 & 226 & 30 & 13 & 664 & & 250 & 328 & 75 \\
\hline$\%$ de cada estadio & 41 & 18 & 35 & 5 & 2 & & & & & \\
\hline
\end{tabular}


Tabla 2.- Listado de las especies y densidad absoluta de sus poblaciones en cada estación muestreada (T: número total de ejemplares; P: primavera: V: verano; O: otoño; I: invierno; D: estadio deutoninfal; P: estadio protoninfal; L: estadio larval).

Table 2.- List of species, seasonal absolute density of Mesostigmata populations (P: spring; V: summer; O: autumn; I: winter; T: total number of specimens; D: deutonymphal stage; P: protonymphal stage; L: larval stage).

\begin{tabular}{|c|c|c|c|c|c|c|c|c|c|c|c|c|c|c|c|c|c|c|c|c|c|c|}
\hline \multirow[t]{2}{*}{ MESOSTIGMATA } & \multicolumn{5}{|c|}{$\mathbf{P}$} & \multicolumn{6}{|c|}{$\mathbf{V}$} & \multicolumn{5}{|c|}{$\mathbf{O}$} & \multicolumn{6}{|c|}{ I } \\
\hline & 우 & $\sigma^{\pi}$ & $\mathrm{D}$ & P L & $\mathrm{T}$ & 우 & $\sigma^{\pi}$ & $\mathrm{D}$ & $\mathrm{P}$ & $\mathrm{L}$ & $\mathrm{T}$ & Q & $\sigma^{7}$ & $\mathrm{D}$ & P L & $\mathrm{T}$ & 우 & $\sigma^{7}$ & $\mathrm{D}$ & $\mathrm{P}$ & $\mathrm{L}$ & $\mathrm{T}$ \\
\hline Anthoseius pirianykae & & & & & 0 & 1 & & & & & 1 & & & & & 0 & & & & & & 0 \\
\hline Arctoseius minutus & 1 & & & & 1 & & & & & & 0 & & & & & 0 & 13 & 2 & & & & 15 \\
\hline Arctoseius venustulus & & & & & 0 & & & & & & 0 & & & & & 0 & 3 & 1 & & & & 4 \\
\hline Cilliba cassidea & & & & & 0 & & 2 & 6 & & 4 & 12 & & & & & 0 & & 1 & 3 & 4 & 4 & 12 \\
\hline Dinychus hispanicus & & & & & 0 & & & & & & 0 & & & & & 0 & & & 1 & 2 & & 3 \\
\hline Holoparasitus calcaratus & & & & & 0 & 2 & & & & & 2 & & & & & 0 & & & & & & 0 \\
\hline Holoparasitus stramenti & 4 & 3 & & & 7 & 3 & 2 & 2 & & & 7 & & & & & 0 & 1 & & & & & 1 \\
\hline Macrocheles montanus & & 1 & 2 & & 3 & & & & & & 0 & & & & & 0 & & & & & & 0 \\
\hline Macrolaspis sp. & & & & & 0 & & & & & & 0 & & & & & 0 & 1 & & & & & 1 \\
\hline Orthadenella lawrencei & & & & & 0 & 1 & 2 & & & & 3 & 2 & & & & 2 & 1 & & & & & 1 \\
\hline Pachylaelaps longisetis & 1 & & & & 1 & & & & & & 0 & & & & & 0 & & 1 & & & & 1 \\
\hline Pachyseiulus ignacii & & & & & 0 & 2 & 2 & & & & 4 & 2 & 2 & & & 4 & & & & & & 0 \\
\hline Pachyseius morenoi & 4 & & & & 4 & 1 & & & & & 1 & 1 & & & & 1 & & & & & & 0 \\
\hline Paragamasus navarrensis & 6 & 2 & 2 & & 10 & 5 & 6 & 10 & & & 21 & 2 & 1 & 1 & 1 & 5 & 2 & 3 & & & & 5 \\
\hline Paragamasus rothamstedensis & 8 & 2 & & & 10 & 8 & 8 & & & & 16 & 2 & & & & 2 & 7 & 4 & & & & 11 \\
\hline Paragamasus ponantinus & & & & & 0 & 1 & 5 & & & & 6 & 7 & 3 & & & 10 & 20 & 19 & & & & 39 \\
\hline Paragamasus sp. 1 & & & & & 0 & 2 & & & & & 2 & 1 & & & & 1 & 1 & & & & & 1 \\
\hline Paragamasus sp. 2 & 1 & 1 & 1 & & 3 & & & & & & 0 & & & & & 0 & 1 & & & & & 1 \\
\hline Paragamasus sp. 3 & & & & & 0 & & & & & & 0 & & & & & 0 & 2 & & & & & 2 \\
\hline Parasitidae immature & & & 46 & & 46 & & & 67 & & & 67 & & & & & 0 & & & 6 & 1 & & 7 \\
\hline Parasitus kraepelini & 10 & 2 & & & 12 & 1 & & & & & 1 & & & & & 0 & 3 & 1 & 1 & 1 & 1 & 7 \\
\hline Parasitus sp. 1 & 1 & & 1 & & 2 & & & & & & 0 & 2 & & 2 & & 4 & 3 & 1 & & & & 4 \\
\hline Pergamasus crassipes & 3 & 5 & 10 & 2 & 20 & 4 & 1 & 5 & & & 10 & & & 1 & & 1 & 1 & & & 1 & & 2 \\
\hline Pergamasus robustus & & & & & 0 & 1 & 5 & & & & 6 & & & & & 0 & & 2 & & & & 2 \\
\hline Pergamasus sp. & & & & & 0 & & & & & & 0 & & 1 & 1 & & 2 & & & & & & 0 \\
\hline Poecilochirus necrophori & & & & & 0 & & & 1 & & & 1 & & & & & 0 & & & & & & 0 \\
\hline Polyaspinus cylindricus & 2 & & 1 & & 3 & 3 & & & 1 & & 4 & & & & & 0 & 6 & & 9 & 2 & 1 & 18 \\
\hline Proctolaelaps robustus & 1 & & & & 1 & & & & & & 0 & & & & & 0 & & & & & & 0 \\
\hline Prozercon davidi & & & & & 0 & 12 & 7 & 2 & & 12 & 22 & & & & & 0 & & & & & & 0 \\
\hline Pseudolaelaps doderoi & & & & & 0 & 3 & & & & & 3 & & & & & 0 & & & & & & 0 \\
\hline Rhodacarus coronatus & 1 & & & & 1 & 3 & & & & & 3 & 15 & & & & 15 & 19 & & & & & 19 \\
\hline Trachytes aegrota & & & & & 0 & 1 & & & & & 1 & & & & & 0 & & & & & & 0 \\
\hline Trachytes welbourni & 1 & 1 & 3 & & 5 & 1 & 3 & & & & 4 & & & & & 0 & 7 & 5 & 5 & 2 & & 19 \\
\hline Uropoda misella & & & 1 & & 1 & 1 & 2 & & & & 3 & & & & & 0 & 1 & & 5 & & & 6 \\
\hline Uropodina sp. 1 & & & 1 & & 1 & & & & & & 0 & & & & & 0 & & 1 & 1 & & & 2 \\
\hline Veigaia nemorensis & & & & & 0 & 1 & & & & & 1 & & & & & 0 & 22 & 12 & 25 & 1 & & 49 \\
\hline Veigaia planicola & 4 & & 9 & & 13 & 3 & 2 & 2 & & & 7 & & & 1 & & 1 & & & & & & 0 \\
\hline Veigaia sanamamedi & & & & & 0 & & & & & & 0 & & & & & 0 & 1 & & & & & 1 \\
\hline Zercon arcuatus & 3 & 3 & 4 & & 10 & & & & & & 0 & & & & & 0 & 8 & & 1 & & 1 & 10 \\
\hline Zercon guadarramicus & 1 & 1 & & & 2 & & & & & & 0 & & & & & 0 & & & & & & 0 \\
\hline Zercon parivus & 3 & & & & 3 & 2 & 1 & & & & 3 & & & & & 0 & 1 & 1 & & & & 2 \\
\hline Zercoseius spathuliger & & & & & 0 & & & & & & 0 & & 1 & & & 1 & & & & & & 0 \\
\hline
\end{tabular}




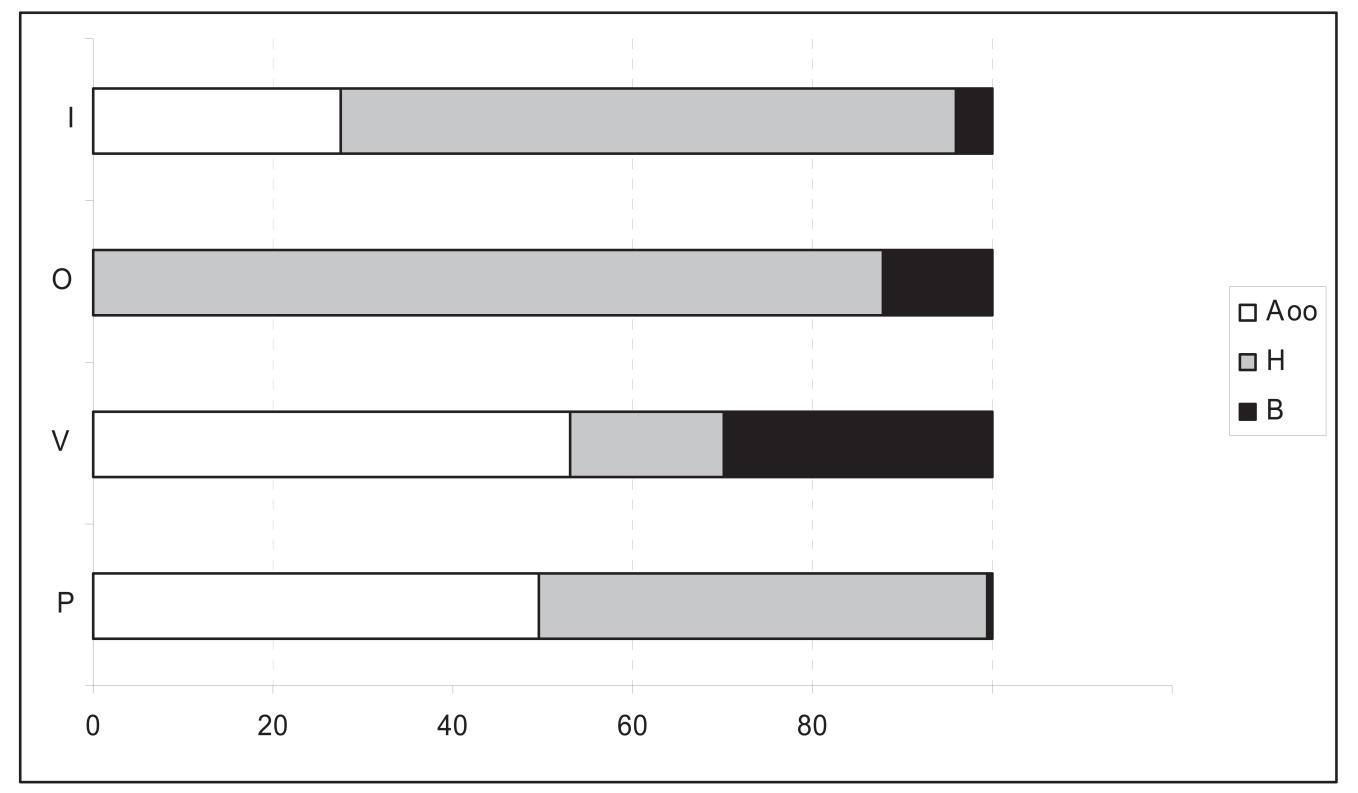

Fig. 1.- Distribución vertical en el suelo del hayedo del número de individuos de los Mesostigmata según las muestras estacionales (Aoo: hojarasca; H: mantillo; B: suelo mineral; P: primavera; V: verano; O: otoño; I: invierno).

Fig. 1.- Vertical distribution in beech forest soil of the number of mesostigmatid mites following the seasonal samples (Aoo: leaf litter; H: humus; B: mineral soil; P: spring; V: summer; O: autumn; I: winter).

La diversidad real de la comunidad se calculó mediante el índice de Shannon-Wiener:

$$
\mathrm{H}^{\prime}=-\sum P_{\mathrm{i}} \log _{2} P_{\mathrm{i}}
$$

donde $P_{\mathrm{i}}$ es la abundancia relativa de la especie $i$.

La equitabilidad o relativa diversidad $\left(J^{\prime}\right)$, es la relación entre la diversidad real $\left(H^{\prime}\right)$ y la máxima diversidad de cada muestra.

Las especies citadas por primera vez para la fauna ibérica aparecen con un asterisco en la Tabla 1.

Los ácaros Mesostigmata se encuentran depositados en la Acarology Collection (Acarology laboratory) of The Ohio State University (Columbus, Ohio, USA) (OSAL).

\section{Resultados}

Debido a que los datos obtenidos de una simple muestra estacional podrían no ser representativos de las comunidades que representarán al hayedo, los resultados que se dedujeran de ellos serían arbitrarios. Dicho esto, debemos de tomar los resulta- dos que hacen referencia a las dinámicas o fluctuaciones estacionales de la comunidad con las debidas precauciones, entendiendo estos como resultados puntuales temporal y espacialmente.

COMPOSICIÓN, ESTRUCTURA Y DIVERSIDAD DE LA COMUNIDAD DE MESOSTIGMATA. Las muestras que se han tomado en el suelo del hayedo de Irati albergan un total de 41 especies of Mesostigmata (Tablas 1 y 2), representadas por 653 ejemplares. Solo 6 especies tienen una abundancia relativa $\geq 5 \%$ y 21 son consideradas como raras (abundancia $<1 \%$ ). Las especies más abundantes en el material recogido son Paragamasus ponantinus (8,3\%), Veigaia nemorensis $(7,5 \%)$, Paragamasus rothamstedensis $(6 \%)$ y Rhodacarus coronatus (6\%).

Más del $51 \%$ de los ejemplares de la comunidad pertenece a la familia Parasitidae (12 especies). La presencia, y en ocasiones su elevada abundancia, de estadios inmaduros de esta familia en todas las muestras procedentes de las cuatro estaciones climáticas (Tabla 2), nos permitiría deducir la existencia de una actividad reproductora constante que mantiene la abundancia de sus poblaciones durante todo el año (Ageeva, 1995). 


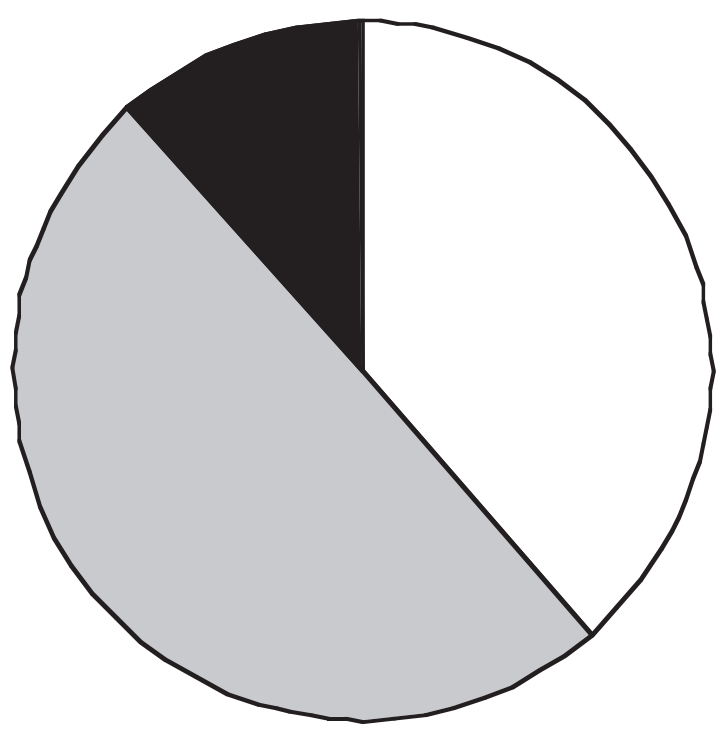

Fig. 2.- Distribución vertical en el suelo del hayedo del número de individuos de los Mesostigmata considerando la muestra global (Aoo: hojarasca; H: mantillo; B: suelo mineral).

Fig. 2.- Vertical distribution in beech forest soil of the number of specimens of mesostigmatid specimens considering the overall sample (Aoo: leaf litter; H: humus; B: mineral soil).

Otras especies, como Prozercon davidi que aparece en las muestras de verano con una abundancia relativa de $11 \%, R h$. coronatus en otoño (31\%) y $V$. nemorensis (20\%) en invierno, pueden ser dominantes sólo durante sus periodos reproductores y son las responsables de los cambios estructurales que sufre la comunidad (Ageeva, 1995).
En relación a la distribución vertical del número de individuos en el suelo (Fig. 1, tabla 5), observamos la siguiente dinámica: en las muestras tomadas en la primavera los ácaros viven igualmente distribuidos en la hojarasca y el mantillo, y solo un pequeño porcentaje de la comunidad habita el suelo mineral; en verano, periodo de utilización del agua almacenada en el suelo, parece que han

Tabla 3.- Índices de diversidad de la comunidad de Mesostigmata del hayedo de Irati (P: primavera: V: verano; O: otoño; I: invierno)

Table 3.- Diversity indexes of the mesostigmatid mite community from Irati beech forest (P: spring; V: summer; O: autumn; I: winter).

\begin{tabular}{lccccc}
\hline Índices de diversidad & $\mathbf{P}$ & $\mathbf{V}$ & $\mathbf{O}$ & I & Global \\
\hline Taxones & 22 & 26 & 13 & 28 & 42 \\
Ejemplares / 25 cm $\mathrm{cm}^{2}$ & 159 & 211 & 49 & 245 & 664 \\
Dominancia D & 0,696 & 0,673 & 0,691 & 0,737 & 0,767 \\
Shannon H' base 2 & 3,580 & 3,649 & 3,055 & $\mathbf{3 , 9 0 5}$ & $\mathbf{4 , 4 2 1}$ \\
Equitabilidad J' & 0,803 & 0,776 & $\mathbf{0 , 8 2 6}$ & 0,812 & $\mathbf{0 , 8 2 0}$ \\
\hline
\end{tabular}


Tabla 4.- Parámetros físico-químicos de las muestras (P: primavera: V: verano; O: otoño; I: invierno).

Table 4.- Physical-Chemical parameters of the samples (P: spring; V: summer; O: autumn; I: winter).

\begin{tabular}{lcccccccc}
\hline & $\mathbf{p H ~ A o o}+\mathbf{H}$ & $\mathbf{p H ~ B}$ & $\mathbf{T}^{\mathbf{a}}$ superficial & $\mathbf{T}^{\mathbf{2}} \mathbf{2 0} \mathbf{~ c m}$ & \% agua Aoo & \% agua $\mathbf{H}$ & \% agua B & \% agua medio \\
\hline $\mathbf{P}$ & 4,64 & 4,26 & 11,70 & 11,50 & 40 & 46 & 25 & 36 \\
$\mathbf{V}$ & 4,09 & 4,28 & 14,00 & 12,90 & 26 & 33 & 25 & 28 \\
$\mathbf{O}$ & 4,46 & 4,87 & 9,00 & 8,00 & 29 & 31 & 23 & 25 \\
$\mathbf{I}$ & 5,87 & 5,91 & 4,10 & 2,70 & 72 & 61 & 36 & 52 \\
\hline
\end{tabular}

emigrado a las capas más profundas; en las muestras de otoño los Mesostigmata han aparecido preferentemente en el mantillo que se encuentra protegido por la nueva capa de hojarasca, capa que parece ya colonizada por los ácaros en invierno. Al considerar todas las muestras como una unidad, se observa que el $50 \%$ de los efectivos de la comunidad prefieren habitar la capa humificada del suelo, el mantillo (Fig. 2, tabla 5).

En cuanto a la demografía de la comunidad, el $41 \%$ de los ejemplares ( $28 \%$ en primavera y otoño - $68 \%$ en la muestra de invierno) corresponden a hembras adultas en comparación con el 18\% (13\% en primavera - $20 \%$ en verano) de machos. El $42 \%$ ( $15 \%$ en otoño - $53 \%$ en primavera) de los ejemplares de la comunidad son inmaduros.

El escaso número de larvas y protoninfas podría deberse al hecho de que estos estadios son más

Tabla 5.- Distribución vertical de los individuos de Mesostigmata en el suelo del hayedo (Aoo: hojarasca; H: mantillo; B: suelo mineral; P: primavera; V: verano; O: otoño; I: invierno).

Table 5.- Vertical distribution in beech forest soil of the number of mesostigmatid specimens (Aoo: leaf litter; H: humus; B: mineral soil; P: spring; V: summer; O: autumn; I: winter).

\begin{tabular}{lccccc}
\hline & P & V & O & I & Totales \\
\hline Aoo & 79 & 103 & 0 & 68 & 250 \\
H & 79 & 33 & 43 & 170 & 328 \\
B & 1 & 58 & 6 & 10 & 75 \\
Total & 159 & 194 & 49 & 248 & 653 \\
\hline
\end{tabular}

vulnerables a la desecación y podrían morir antes de escapar de la muestra en el proceso de su extracción.

Al observar los datos estacionales de los índices de diversidad de esta comunidad (Tabla 3), nos encontramos con los máximos valores de diversidad $H^{\prime}$ en invierno $\left(H^{\prime}\right.$ es 3,9$)$. Los valores mínimos de abundancia, riqueza y diversidad en otoño $\left(H^{\prime}\right.$ es 3,05$)$ y los mínimos de equitabilidad $\left(J^{\prime}\right)$ encontrados en verano, coinciden con el menor porcentaje de agua en la muestra $(25-28 \%$ de agua) (Tabla 4). El hecho de que la humedad relativa del suelo debe ser reconocida como el factor limitante para los ácaros del suelo ya fue puesto de manifiesto por otros autores (Salmane, 2000).

La equitabilidad (rango 0-1) es una medida del balance poblacional en las comunidades. El valor global de $J^{\prime}$ calculado para todas las muestras tomadas es 0,82 , lo que nos informa de un buen balance poblacional para esta comunidad (Daget, 1979), con una alta uniformidad en la densidad de las especies (Magurran 1988).

\section{Conclusiones}

La comunidad de ácaros Mesostigmata en este hayedo es muy diversa $\left(H^{\prime}\right.$ es 4,42$)$, uniforme y estable $\left(J^{\prime}\right.$ es 0,82$)$ cuando se compara con otras comunidades de ácaros de otros hábitat de la Península (Arroyo, 2005) y con otros bosques naturales del Norte de España (Jordana et al., 1987; Iturrondobeitia et al., 1985; Iturrondobeitia \& Saloña, 1992a, 1992b; Iturrondobeitia et al., 1997; Moraza, 2006). En este hayedo la humedad relativa del suelo, entre otros factores ecológicos, parece ser la causa de las variaciones de diversidad y estabilidad de los Mesostigmata. Se citan por primera vez para la fauna ibérica 19 especies. 


\section{Referencias}

Ageeva, T. Z., 1995. Seasonal dynamics of Mesostigmata (Parasitiformes) in soils of urban Moscow oak forest. Izvestiya Akademii Nauk Seriya Biologicheskaya, 6: 722-728.

AMORES, G., 2002. Evaluación del estado fitosanitario y de contaminación de los hayedos de la Comunidad Foral de Navarra mediante el empleo de indicadores. Tesis Doctoral. Universidad de Navarra. Pamplona. 332 pp.

Arbea, J. J. \& Jordana, R., 1988. Efecto de la repoblación con alerce (Larix kaempferi) en la zona norte de Navarra, sobre la estructura de la poblaciones de colembolos edáficos. In: Iturrondobeitia J. C. (ed.). Biologia ambiental. Actas del Congreso de Biologia Ambiental (2 Congreso Mundial Vasco), Tomo 2. Servicio Editorial de la Universidad del País Vasco: 253-265.

Ariño, A. H., Moraza, M. L., Hernandez, M. A. \& JordANA, R., 1992. Cambios en la edafofauna de hayedos alterados. Actas del Congreso Internacional del haya. R. E. Rosselló (Ed.). Investigación Agraria: C 060-C 066.

Arroyo, J., Iturrondobeita, J. C., Rad, C. \& GonzÁlez CARCedo, S., 2005. Oribatid mite (Acari) community structure in steppic habitats of Burgos Province, central northern Spain. Journal of Natural History, 39(39): 3453-3470.

Buryn, R., 1992. Gamasid fauna (Acari, Mesostigmata) of a hedge and adjacent meadow in upper franconia (Bavaria, Germany). Pedobiologia, 36(2): 97-108.

DAGET, J., 1979 Les modéles mathématiques en écologie. Masson (Collection d'écologie; 8). Paris. 172 pp.

Iturrondobeitia, J. C. \& Saloña, M. I., 1985. Contribución al estudio sinecológico de los acaros oribatidos de varios ecosistemas vizcainos (Acarida, Oribatida). Actas do Congresso Iberico de Entomologia, 2(3): 353-362.

Iturrondobeitia, J. C. \& SAloña, M. I., 1992a. Estudio de las comunidades de oribatidos (Acari, Oribatei) de varios ecosistemas de Vizcaya y una zona próxima; 4. Relación entre fauna y factores del suelo. Revue d'Ecologie et de Biologie du Sol, 28(4): 443-459.

Iturrondobeitia, J. C. \& SAloñA, M. I., 1992b. Relationship between oribatids and physico-chemical soil factors. In: Teller, A., Mathy, P. \& Jeffers, J. N. R. (eds). Responses of forest ecosystems to environmental changes. Proceedings of the First European Symposium on Terrestrial Ecosystems, Forests and Woodland. Elsevier Applied Science. London \& New York: 969-971.
Jordana, R., Arbea, J. I., Moraza, L., Montenegros, E., Mateo, M. D., Hernandez, M. A. \& Herrera, L. 1987. Effect of reafforestation by conifers in natural biotopes of middle and South Navarra (Northern Spain). Revue suisse de Zoologie, 94(3): 491-502.

Magurran, A., 1988. Diversidad ecológica y su medición. Vedrá. Barcelona. 200 pp.

Moraza, M. L., 1982. Ácaros Oribátidos de los hayedos de Navarra. Tesis Doctoral. Universidad de Navarra. Pamplona. 666 pp.

MorazA, M. L., 2006. Efecto de la degradación de un encinar de Quercus rotundifolia en la comunidad de ácaros Cryptostigmados y Mesostigmados (Acari: Cryptostigmata, Mesostigamata). Revista Ibérica de Aracnología, 13: 173-184.

MorazA, M. L., Herrera, L. \& Pérez-IÑIgo, C., 1980. Estudio faunístico del Macizo de Quinto Real. I. Acaros Oribátidos (Acari, Oribatei). Publicaciones de Biología, Universidad de Navarra, 1: 1-30.

SAlmane, I., 2000. Investigation of the seasonal dynamics of soil Gamasina mites (Acari: Mesostigmata) in Pinaceum myrtilorum, Latvia. Ekológia (Bratislava), 19: 245-252.

USHER, M. B., 1971. Seasonal and vertical distribution of a population of soil arthropods - Mesostigmata. Pedobiología, 11(1): 27-39.
Recibido, 31-X-2006 Aceptado, 29-III-2007 Publicado, 27-VI-2007 